\title{
A Simple Optimal Power Flow Model with Energy Storage
}

\author{
K. Mani Chandy, Steven H. Low, Ufuk Topcu and Huan Xu
}

\begin{abstract}
The integration of renewable energy generation, such as wind power, into the electric grid is difficult because of the source intermittency and the large distance between generation sites and users. This difficulty can be overcome through a transmission network with large-scale storage that not only transports power, but also mitigates against fluctuations in generation and supply. We formulate an optimal power flow problem with storage as a finite-horizon optimal control problem. We prove, for the special case with a single generator and a single load, that the optimal generation schedule will cross the time-varying demand profile at most once, from above. This means that the optimal policy will generate more than demand initially in order to charge up the battery, and then generate less than the demand and use the battery to supplement generation in final stages. This is a consequence of the fact that the marginal storage cost-to-go decreases in time.
\end{abstract}

\section{INTRODUCTION}

The optimal power flow (OPF) problem is to optimize a certain objective over power network variables under certain constraints. The variables may include real and reactive power outputs, bus voltages and angles; the objective may be the minimization of generation cost or maximization of user utilities; and the constraints may be bounds on voltages or power levels, or that the line loading not exceeding thermal or stability limits. The OPF has been studied for over half a century since the pioneering work of Carpentier [1]; see surveys for example in [2], [3], [4], [5], [6] and [7]. Its history is roughly a continuous application of more and more sophisticated optimization techniques [7]. Most of the models involve static optimization as, without large scale storage, power supply and demand must be matched exactly at all times, and therefore OPF can be solved in isolation from one period to the next. In this paper, we formulate a simple OPF model with storage and study how storage allows optimization of power generation across multiple time periods.

The model is motivated by the intensifying trend to deploy renewable energy such as wind or solar power. In the state of California, peak demand for power in 2003 reached 52 GW, with projections for the year 2030 exceeding $80 \mathrm{GW}$ [8], [9]. With fossil fuel and nuclear plants retiring in the next few decades, and a required $15 \%$ reserve margin, an additional 60 GW of new generation capacity will be needed by 2030 [9]. In 2006, Southern California Edison, the primary electricity

The authors are with California Institute of Technology, Pasadena, CA, 91125, USA. K. M. Chandy is with the Department of Computer Science; S. H. Low is with the Department of Computer Science and the Department of Electrical Engineering; U. Topcu is with the Department of Control and Dynamical Systems; and $\mathrm{H} . \mathrm{Xu}$ is with the Department of Mechanical Engineering. Email addresses: manidcaltech.edu, slowecaltech.edu, utopculcds. caltech. edu, mumudcaltech.edu. utility company for southern California, signed a contract to provide $1.5 \mathrm{GW}$ of power to its customers from wind projects in the Tehachapi area in California [10]. Not only is renewable energy more environmentally friendly, but its potential is high. It has been estimated that if $20 \%$ of the energy that could be harvested from wind farms across the globe were used, all of the world's electricity demands could be met several times over [11]. There are however two major challenges in integrating wind or solar power into the current system. First, their output fluctuates widely, rapidly, and randomly, a great operational hurdle. Second, the geographical locations of wind or solar farms are inevitably far from the load centers. These two problems calls for largescale storage that can absorb short-term fluctuations and transmission capacity that not only transports power from generation to load, but can also provide spatial diversity in generation to mitigate intermittency of renewable sources. See, for instance, [12] for an early simulation study on how battery can help with regulation and peak-shaving in economic dispatch. References [13], [14], [15] investigate the utility of energy storage in the integration of renewable energy resources and the concept of microgrids.

The model studied in this paper does not capture the full spectrum of issues involved in the integration of renewable sources, but only focuses on the effect of storage to optimal power flow. For instance, it assumes time-varying but deterministic, as opposed to stochastic, generation and demand. Moreover, the calculation of the optimal power flow assumes the full knowledge of the demand schedule. In practice, demands are typically estimated 24 hours in advance to an accuracy of a few percentage points.

The classical OPF problem without storage is a static optimization as the need to balance supply and demand at all times decouples the optimization in different periods. The addition of storage introduces correlation, and an opportunity to optimize, across time, e.g., charge when (and where) the cost of generation is low and discharge when it is high. Our model characterizes the structure of optimal generation and charge/discharge schedule. In Section II, we formulate the OPF model with storage as a finite-horizon optimal control problem. In Section III, we consider the special case with a single generator and a single load (SGSL case). Without battery, the generation must be exactly equal to the load in each period. With battery, we prove that the optimal generation schedule will cross the time-varying demand profile at most once, from above. This means that the optimal policy will generate more than demand initially in order to charge up the battery, and then generate less than the demand and use the battery to supplement generation in final stages. This is a 
consequence of the fact that the marginal storage cost-to-go decreases in time. In Section IV, we discuss the optimality conditions for the general network case and present some numerical examples that suggest the intuition in the SGSL case might generalize to the network case.

\section{MODEL AND PROBLEM FORMULATION}

We now present a simple OPF model with energy storage and time-varying generation costs and demands. The model ignores reactive power and makes other simplifying assumptions. Our goal is to understand the impact of storage on optimal generation schedule.

Consider a set $\mathscr{G}$ of generation nodes/buses connected to a set $\mathscr{D}$ of demand nodes/buses by a transmission network. Let $\mathscr{N}=\mathscr{G} \cup \mathscr{D}$ be the set of all nodes. The transmission network is modeled by the admittance matrix $Y$, where $Y_{i j}=Y_{j i}$ is the admittance between nodes $i$ and $j$. If nodes $i$ and $j$ are not directly connected, $Y_{i j}=0$.

The (real) power flow from node $i$ to node $j$ at time $t$ is $V_{i} V_{j} Y_{i j} \sin \left(\theta_{i}(t)-\theta_{j}(t)\right)$ for $i \neq j \in \mathscr{N}$. In this paper, we will assume $\left|\theta_{i}(t)-\theta_{j}(t)\right|$ is small and approximate $\sin \left(\theta_{i}(t)-\theta_{j}(t)\right)$ by $\theta_{i}(t)-\theta_{j}(t)$ [16]. The amount of power delivered over link $(i, j)$ is limited by thermal effects and network stability [17, chapter 4]. Current flows produce power losses alongside heat generation, reducing transmission efficiency and creating line sag from temperature rises. We capture these constraints as

$$
V_{i} V_{j} Y_{i j}\left(\theta_{i}(t)-\theta_{j}(t)\right) \leq \bar{q}_{i j}(t), \quad i \neq j \in \mathscr{N}
$$

where $\bar{q}_{i j}(t)$ represents the line capacity from nodes $i$ to $j$. From Kirchoff's laws, the net power export from node $i$ at time $t$ is given by

$$
q_{i}(t)=\sum_{j \in \mathscr{N}} V_{i} V_{j} Y_{i j}\left(\theta_{i}(t)-\theta_{j}(t)\right), \quad i \in \mathscr{N} .
$$

If $q_{i}(t)$ is positive, node $i$ supplies power at time $t$. Otherwise, it consumes power at time $t$.

Each node $i \in \mathscr{D}$ demands a fixed amount $d_{i}(t)$ of power at time $t$ that must be met by supplies from the generation nodes

$$
q_{i}(t)=-d_{i}(t), \quad i \in \mathscr{D} .
$$

Each generation node $i \in \mathscr{G}$ has both a generator that produces $g_{i}(t)$ amount of power at time $t$ and a battery that can charge or discharge $r_{i}(t)$ amount of power at time $t$. The net power export $q_{i}(t)$ from generator node $i$ at time $t$ consists of the power from the node's generator and battery:

$$
\begin{aligned}
& q_{i}(t)=g_{i}(t)+r_{i}(t), \quad i \in \mathscr{G} \\
& g_{i}(t) \geq 0, \quad i \in \mathscr{G}
\end{aligned}
$$

Note that $r_{i}(t)$ can either be negative (battery is charging) or positive (battery is discharging). The battery energy level $b_{i}(t)$ at node $i \in \mathscr{G}$ evolves according to ${ }^{1}$

$$
b_{i}(t)=b_{i}(t-1)-r_{i}(t), \quad i \in \mathscr{G}
$$

\footnotetext{
${ }^{1}$ In this paper, all power quantities such as $g_{i}(t), q_{i}(t), r_{i}(t), d_{i}(t)$ are in the unit of energy per unit time, so the energy produced/consumed in time period $t$ are $g_{i}(t), q_{i}(t), r_{i}(t), d_{i}(t)$, respectively.
}

with given initial energy level $b_{i}(0) \geq 0$. Battery storage is bounded by a minimum and a maximum capacity

$$
0 \leq b_{i}(t) \leq B_{i} \quad i \in \mathscr{G}
$$

We assume the cost $c_{i}\left(g_{i}, t\right)$ of generation at generator $i \in \mathscr{G}$ is a function of the amount of generated power $g_{i}$ and time $t$. There is a battery cost $h_{i}\left(b_{i}, r_{i}\right)$ as a time-invariant function of energy level $b_{i}$ and the power draw $r_{i}$. Finally, there is a terminal cost $h_{i}^{T}\left(b_{i}(T)\right)$ on the final battery energy level $b_{i}(T)$.

The classical OPF problem without storage determines the voltage angles $\theta_{i}(t), i \in \mathscr{N}$, and power generations $q_{i}(t), i \in \mathscr{G}$, so as to minimize the total generation cost. Without storage, there is no correlation across time, and therefore the optimization problem is static and can be solved in isolation from one period to the next. The transmission network $Y$ allows optimization across space. Storage allows optimization across time as well, i.e., charge when (and where) the cost of generation is low and discharge when it is high. The optimization problems in each period thus become coupled, yielding an optimal control problem.

Given admittance matrix $Y$, initial battery levels $b_{i}(0) \geq 0$ and storage capacities $B_{i} \geq 0$ for $i \in \mathscr{G}$, link capacities $\bar{q}_{i j}$ for $i, j \in \mathscr{N}$, and demand profiles $d_{i}(t)$ for $i \in \mathscr{D}, t=1, \ldots, T$, the OPF problem with energy storage is

\section{OPF-S}

$$
\begin{aligned}
& \min \sum_{i \in G_{t}} \sum_{t=1}^{T}\left(c_{i}\left(g_{i}(t), t\right)+h_{i}\left(b_{i}(t), r_{i}(t)\right)\right) \\
& +\sum_{i \in \mathscr{G}} h_{i}^{T}\left(b_{i}(T)\right) \\
& \text { over } \quad \theta, q, r, g, b \\
& \text { s.t. } \quad(1),(2),(3),(4),(5),(6),(7) \text {, }
\end{aligned}
$$

where $t=1, \ldots, T$ in constraints (1)-(7). Note that in this model only the generation costs $c_{i}\left(g_{i}, t\right)$ and demand profiles $d_{i}(t)$ are time-varying.

In this paper, we restrict ourselves to the quadratic costs

$$
c_{i}\left(g_{i}(t), t\right):=\frac{1}{2} \gamma_{i}(t) g_{i}^{2}(t)
$$

where $\gamma_{i}(t)$ models the time-varying nature of the generation costs. The convexity of $c_{i}$ in $g_{i}$ reflects the decreased efficiency of the generator when producing very high amounts of power [17, chapter 11]. We also assume a battery cost that is independent of the power draw $r_{i}$, but dependent only on the energy level $b_{i}$, i.e.,

$$
h_{i}\left(b_{i}, r_{i}\right)=h_{i}\left(b_{i}\right) \text {. }
$$

For example, if it is desirable to maintain a full battery level at the end of each period, then a cost function $h_{i}\left(b_{i}\right)=$ $\alpha_{i}\left(B_{i}-b_{i}\right)$ for some $\alpha_{i}>0$, imposes a penalty proportional to the deviation from its capacity.

Notations. We will use $h^{\prime}(b(t))$ and $\frac{d h}{d b}(b(t))$ interchangeably. For both primal variables, such as $g_{i}(t), b_{i}(t)$, and their dual variables, such as $\tilde{b}_{i}(t), \underline{b}_{i}(t), \bar{b}_{i}(t)$, a ${ }^{*}$ denotes their value at optimality, e.g., $g_{i}^{*}(t), b_{i}^{*}(t), \tilde{b}_{i}^{*}(t), \underline{b}_{i}^{*}(t), \bar{b}_{i}^{*}(t)$. For $x \in \mathbb{R},[x]^{+}:=\max (x, 0)$. 


\section{Single Generator Single LOAD (SGSL) CASE}

In this section we solve the simplest case of a single generator (with a battery) connected to a single load. Removal of the network structure allows us to completely characterize the solution and make transparent the effect of storage on the optimal generation schedule and the optimal chargedischarge schedule of the battery. We expect the basic insight to generalize to the network case, the discussion of which is in the next section.

\section{A. SGSL model}

From (2), (3), and (4) we have

$$
g(t)+r(t)=d(t)
$$

This simplifies the problem OPF-S into the following

$$
\begin{array}{cl}
\min & \sum_{t=1}^{T}(c(g(t), t)+h(b(t)))+h^{T}(b(T)) \\
\text { over } & g(t), b(t) \\
\text { s. t. } & b(t)=b(t-1)-d(t)+g(t) \\
& g(t) \geq 0 \\
& b(t) \geq 0 \\
& B-b(t) \geq 0 \\
\text { for } & t=1, \ldots, T
\end{array}
$$

where $b(0) \geq 0$ is given, and the cost functions $c$ and $h$ are given by (11) and (12) respectively. The capacity constraint (1) degenerates into $d(t) \leq \bar{q}$ where $\bar{q}$ is the capacity of the link connecting the generator to the load. For feasibility, we assume that this constraint is satisfied. For $t=1, \ldots, T$, let $\tilde{b}(t)$ be the dual variables associated with constraints (14), $\hat{\lambda}(t)$ with constraints $(15), \underline{b}(t)$ with constraints $(16), \bar{b}(t)$ with constraints (17).

The problem OPF-S is a convex program and therefore the Karush-Kuhn-Tucker (KKT) condition is both necessary and sufficient for optimality. Differentiating with respect to $b(t)$, the KKT condition implies

$$
\frac{d h}{d b}\left(b^{*}(t)\right)+\tilde{b}^{*}(t)-\tilde{b}^{*}(t+1) \mathbf{1}(t<T)=\underline{b}^{*}(t)-\bar{b}^{*}(t)
$$

where 1 denotes the indicator function. This defines a recursion on $\tilde{b}^{*}(t)$ whose solution, for $t=1, \ldots, T$, is

$$
\tilde{b}^{*}(t)=H^{*}(t)+B^{*}(t)
$$

where

$$
\begin{aligned}
H^{*}(t) & :=\sum_{\tau=t}^{T}-\frac{d h}{d b}\left(b^{*}(\tau)\right)-\frac{d h^{T}}{d b}\left(b^{*}(T)\right) \\
B^{*}(t) & :=\sum_{\tau=t}^{T}\left(\underline{b}^{*}(\tau)-\bar{b}^{*}(\tau)\right) .
\end{aligned}
$$

Here, $b^{*}(t) \underline{b}^{*}(t)=0$ and $\left.\left(B-b^{*}(t)\right) \bar{b}^{*}(t)\right)=0$ by complementary slackness. $H^{*}(t)$ is the marginal storage cost-to-go at time $t$. By assumption (see A0 below) $h^{\prime}(b)<0,\left(h^{T}\right)^{\prime}(b)<0$, and hence $H^{*}(t)>0$. For example, if $h(b)=\alpha(B-b)$ and $h^{T}(b)=\alpha_{T}(B-b)$ for some constants $\alpha, \alpha_{T}>0$, then $H^{*}(t)=\alpha(T+1-t)+\alpha_{T}$.
Differentiating the objective function with respect to $g(t)$, the KKT condition implies $(\tilde{b}(t)=\lambda(t))$

$$
\gamma(t) g^{*}(t)=\tilde{b}^{*}(t)+\hat{\lambda}^{*}(t),
$$

where $\hat{\lambda}^{*}(t) g^{*}(t)=0$ by complementary slackness. Combining with (18), the optimal solution is characterized by

$$
\begin{aligned}
& g^{*}(t)=\left[\bar{g}(t)+\frac{B^{*}(t)}{\gamma(t)}\right]^{+} \\
& b^{*}(t)=b^{*}(t-1)-d(t)+\left[\bar{g}(t)+\frac{B^{*}(t)}{\gamma(t)}\right]^{+}
\end{aligned}
$$

where $\bar{g}(t)$, the nominal generation at time $t$, is defined as

$$
\bar{g}(t):=\frac{H^{*}(t)}{\gamma(t)} .
$$

We now derive the structure of the optimal generation schedule $g^{*}$ and the optimal battery energy level $b^{*}$ from (19)-(21).

\section{B. Optimal solution}

It is instructive to first look at the case where the battery constraint is inactive, i.e., $b(t) \in(0, B)$. In this case, $B^{*}(t) \equiv$ 0 , and the nominal generation schedule in (21) is optimal, i.e., $g^{*}=\bar{g}$. This satisfies the condition

$$
\gamma(t) g^{*}(t)=H^{*}(t)=\sum_{\tau=t}^{T}-\frac{d h}{d b}\left(b^{*}(\tau)\right)-\frac{d h^{T}}{d b}\left(b^{*}(T)\right) .
$$

The left-hand side is the marginal generation cost at time $t$ and the right-hand side is the marginal storage cost-to-go from $t$ to $T$. At optimality, one cannot reduce the cost further by increasing (decreasing) generation by a unit and raising (reducing) battery level by the same amount. Moreover, if $h^{\prime}(b)<0$, the marginal storage cost-to-go, and therefore the marginal generation cost, strictly decreases in time under an optimal policy. These intuitions will generalize to the general case when the battery constraints may be active, as we now show.

Our first main result characterizes the optimal generation schedule $g^{*}(t)$ under the assumptions

A0: For $t=1, \ldots, T, d(t)>0, \gamma(t)>0$. For $b \geq 0, h^{\prime}(b)<0$ and $\left(h^{T}\right)^{\prime}(b)<0$;

A1: For $t=1, \ldots, T, \quad \gamma(t) d(t)-\gamma(t+1) d(t+1)<$ $-h^{\prime}(b(t))$.

Assumption A1 restricts how fast the product $\gamma(t) d(t)$ can decrease. If the battery constraint is not active, then the optimal generation $g^{*}$ will balance the marginal generation cost and the marginal storage cost-to-go. Hence, the optimal marginal generation cost $\gamma(t) g^{*}(t)$ will decrease at the same rate at which the marginal storage cost-to-go $H^{*}(t)$ decreases, which is the marginal storage cost $h^{\prime}\left(b^{*}(t)\right)$ at time $t$.

The case where the battery constraint is active is more complicated because the optimal generation must anticipate future starvation and saturation. As we will see below, if the product $\gamma(t) d(t)$ decreases more slowly than marginal storage cost $h^{\prime}(b(t))$, then the optimal generation $g^{*}$ retains 
a simple global structure where $g^{*}$ crosses demand $d(t)$ at most once, from above, and the optimal battery level $b^{*}$ is unimodal. Indeed, under assumption $\mathrm{A} 1$, the optimal generation schedule generally has three phases. In the first phase, $g^{*}(t)>d(t)$ and the battery charges. In the second phase, $g^{*}(t)=d(t)$ and the battery remains saturated at $b^{*}(t)=B$. In the third phase, $g^{*}(t)<d(t)$ and the battery discharges. Degenerate cases where one or more of the phases are missing are possible, depending on problem parameters $T, b(0), \gamma(t)$, and $d(t)$.

If the battery never saturates, the optimal schedule degenerates into (at most) two phases. If the battery does saturate, the optimal schedule has three phases, defined by the beginning time $l+1$ and the end time $m$ of the second phase, with $0<l \leq m \leq T$, where $b^{*}(t)=B$ for $t=l, \ldots, m$. To describe the optimal generation $g^{*}$ precisely, define the following time averages over the entire horizon and over the initial and final phases

$$
\begin{array}{rlrl}
\bar{d} & :=\frac{1}{T} \sum_{t=1}^{T} d(t), & \bar{\gamma}:=\left(\frac{1}{T} \sum_{t=1}^{T} \frac{1}{\gamma(t)}\right)^{-1}, \\
\bar{d}_{1}:=\frac{1}{l} \sum_{t=1}^{l} d(t), & \bar{\gamma}_{1}:=\left(\frac{1}{l} \sum_{t=1}^{l} \frac{1}{\gamma(t)}\right)^{-1}, \\
\bar{d}_{3}:=\frac{1}{T-m} \sum_{t=m+1}^{T} d(t), & \bar{\gamma}_{3}:=\left(\frac{1}{T-m} \sum_{t=m+1}^{T} \frac{1}{\gamma(t)}\right)^{-1} .
\end{array}
$$

Our main result implies that it is optimal to charge the battery initially and discharge it later.

Theorem 1: Suppose A0 and A1 hold and $0<b(0)<$ $B$. The optimal generation $g^{*}$ crosses the demand curve $d(t)$ at most once, from above, and therefore the optimal battery level $b^{*}$ is unimodal. Moreover, they take one of the following two forms.

1) The battery never saturates, and the optimal generation schedule is

$$
g^{*}(t)=\bar{g}(t)+\frac{\bar{\gamma}}{\gamma(t)}[\bar{d}-\sigma]^{+}
$$

with

$$
\sigma:=\frac{1}{T} \sum_{t=1}^{T} \bar{g}(t)+\frac{b(0)}{T} .
$$

Moreover, $b^{*}(T)=T[\sigma-\bar{d}]^{+}$.

2) There exist times $l$ and $m$, with $0<l \leq m \leq T$, for which $b^{*}(t)=B$ for $t=l, \ldots, m$. Before time $l+1$, the battery charges and its stored energy strictly increases over time towards $B$. After time $m$, it discharges and its stored energy strictly decreases over time from $B$. The optimal generation $g^{*}$ has three phases:

$$
g^{*}(t)= \begin{cases}\bar{g}(t)-\frac{\bar{\gamma}_{1}}{\gamma(t)}\left(\sigma_{1}-\bar{d}_{1}\right), & t=1, \ldots, l \\ d(t), & t=l+1, \ldots, m \\ \bar{g}(t)+\frac{\bar{\gamma}_{3}}{\gamma(t)}\left[\bar{d}_{3}-\sigma_{3}\right]^{+}, & t=m+1, \ldots, T\end{cases}
$$

where

$$
\begin{array}{r}
\sigma_{1}:=\frac{1}{l} \sum_{t=1}^{l} \bar{g}(t)-\frac{B-b(0)}{l} \\
\sigma_{3}:=\frac{1}{T-m} \sum_{t=m+1}^{T} \bar{g}(t)+\frac{B}{T-m} .
\end{array}
$$

Moreover, $b^{*}(T)=(T-m)\left[\sigma_{3}-\bar{d}_{3}\right]^{+}$.

Case 1 of Theorem 1 is illustrated in Figure 1. Here,

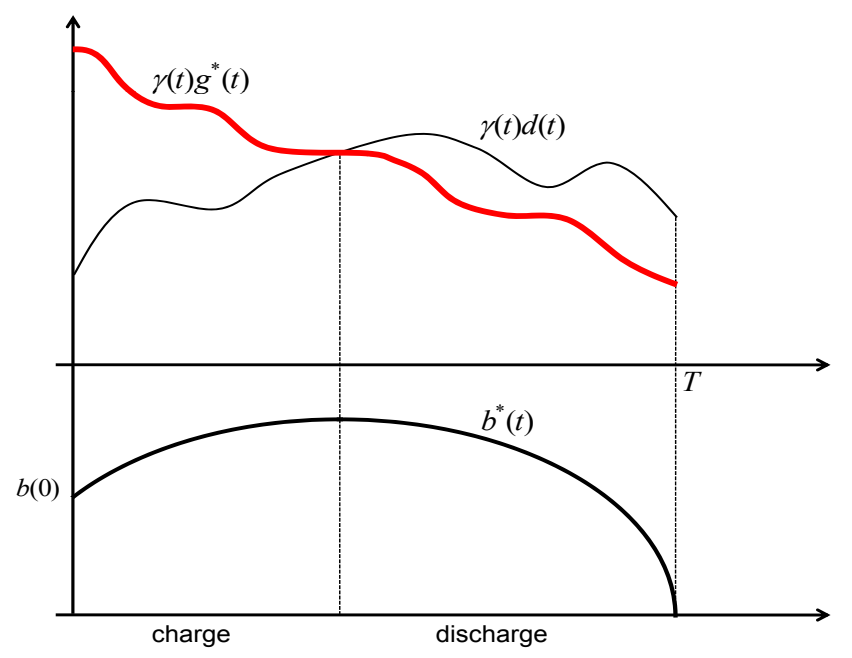

Fig. 1. Optimal marginal generation cost $\gamma(t) g^{*}(t)$, in comparison with $\gamma(t) d(t)$ and battery level $b^{*}(t)$. This figure illustrates case 1 of Theorem 1 with $\bar{d}>\sigma$.

the battery level remains strictly below $B$ at all times. The threshold $\sigma$ is the sum of average nominal generation and the initial battery energy per unit time. Under the nominal generation schedule, the total available energy over the entire period $t=1, \ldots, T$ is $T \sigma$. If the average demand $\bar{d}$ is lower than the threshold $\sigma$, then the nominal generation is optimal, $g^{*}=\bar{g}$, and the terminal battery level at the end of the control horizon is $b^{*}(T)=T(\sigma-\bar{d})$. Otherwise, the nominal generation schedule will deplete the battery level to zero before time $T$, which is not optimal, and hence the generation is shifted up by $\bar{\gamma}(\bar{d}-\sigma) / \gamma(t)$ at each time $t$, so that the battery level hits zero exactly (and only) at time $T$.

Case 2 of Theorem 1 is illustrated in Figure 2. In this case, the battery level rises from $b(0)$ to $B$ in phase 1 , stays at $B$ in phase 2 , and drains in phase 3 . The threshold $\sigma_{3}$ is the sum of the average nominal generation over the third phase and the per-period battery level at the beginning of the third phase. If the nominal generation is used in the third phase, then the total available energy over this phase is $(T-m) \sigma_{3}$. If the average demand $\bar{d}_{3}$ over the third phase is less than the threshold $\sigma_{3}$, then the nominal generation is optimal in the third phase, $g^{*}=\bar{g}$, and the terminal battery level is $b^{*}(T)=$ $(T-m)\left[\sigma_{3}-\bar{d}_{3}\right]^{+}$(this is similar to case 1 with initial battery level $B$, but over only the third phase). On the other hand, if $\bar{d}_{3}>\sigma_{3}$, then the nominal generation in the third phase is shifted up in the third phase so that the battery level hits zero exactly at time $T$. 


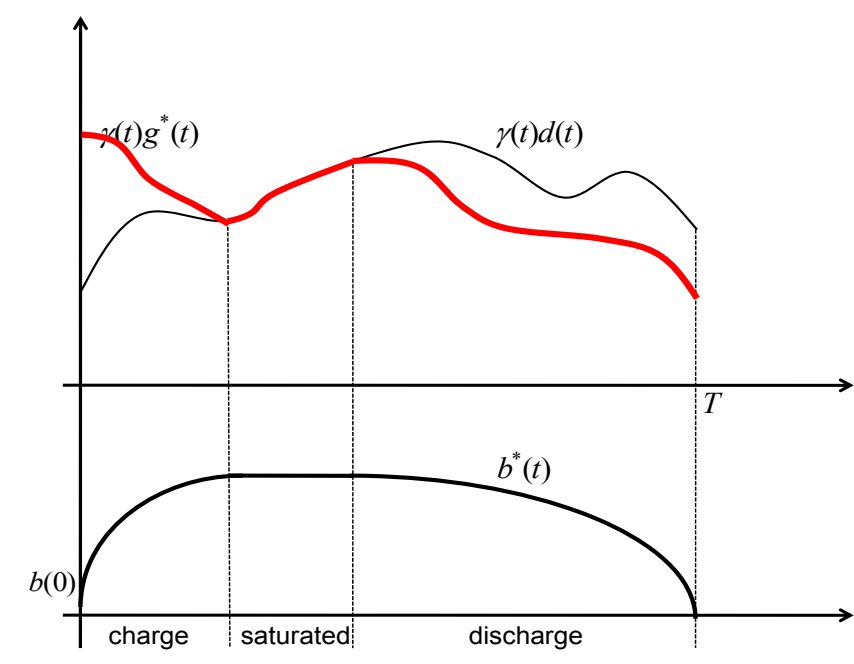

Fig. 2. Optimal marginal generation cost $\gamma(t) g^{*}(t)$, in comparison with $\gamma(t) d(t)$, and battery level $b^{*}(t)$. This figure illustrates case 2 of Theorem 1 with $\bar{d}_{1}<\sigma_{1}$ and $\bar{d}_{3}<\sigma_{3}$.

\section{Example 1: SGSL case without assumption A1.}

We present an SGSL example where assumption A1 does not hold. The horizon $T=24$ hours and each time step $t$ represents 1 hour. The demand profile is

$$
d(t)=10 \cdot \sin \left(\frac{4 \pi}{T-1}(t-1)\right)+50 \mathrm{GJ} .
$$

Battery capacity $B$ is $25 \mathrm{GJ}$ (billion joules), with an initial battery level $b(0)$ of 12.5 GJ. We set $\gamma(t) \equiv 1$ in the generation cost $c$ and use $h(b)=\alpha(B-b)$ with $\alpha=2$. Figure 3 shows the numerical result for one horizon representing a 24hour day. The optimal battery level $b^{*}$ increases to saturation, but because demand decreases faster than that required for Assumption A1, $b^{*}$ discharges and recharges twice more before reaching zero at the final time. The optimal generation $g^{*}$ remains linear (because $h(b)$ is affine and $\gamma$ is timeinvariant) when the battery charges and discharges, and follows demand when the battery is saturated, but does so several times over the entire control horizon.

\section{Proof idea}

In this subsection, we sketch the proof idea by describing some finer properties of the optimal solution. The detailed proofs are omitted due to space constraint.

The first lemma states that, under an optimal policy, the battery is never exhausted until possibly the last period. This is a consequence of the convexity of the cost function $c(g, t)$ in $g$.

Lemma 1: Suppose A0 and A1 hold. Under an optimal policy, $b^{*}(t)>0$ for $t=1, \ldots, T-1$.

The next result describes the behavior in the third phase of Theorem 1. Once an optimal generation $g^{*}(t)$ drops below the demand $d(t)$, it will remain strictly below the (time-varying) demand for subsequent periods. The battery therefore drains. Additionally, the optimal marginal cost $\gamma(t) g^{*}(t)$ decreases at a rate equal to the marginal battery cost $h^{\prime}\left(b^{*}(t)\right)$ at time $t$.

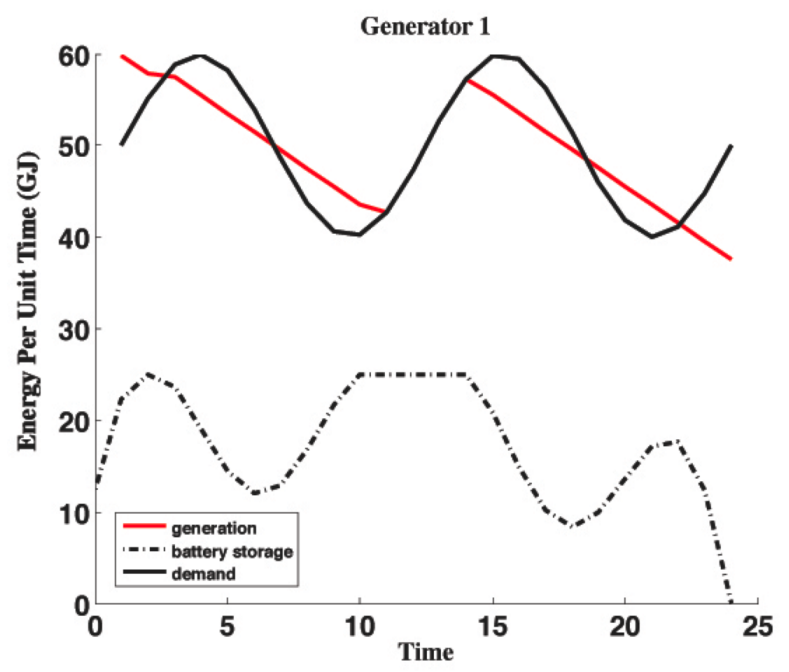

Fig. 3. Numerical results for a single generator single load when assumption A1 does not hold. Optimal generation $g^{*}$ is linear when the battery charges and discharges, and matches demand when the battery is saturated.

Lemma 2: Suppose A0 and A1 hold. If $g^{*}\left(t_{1}\right)<d\left(t_{1}\right)$ for some $t_{1} \in\{1, \ldots, T-1\}$ under an optimal policy, then

1) $0<g^{*}(t)<d(t)$, for $t=t_{1}, \ldots, T$. Moreover, for $t=$ $t_{1}, \ldots, T-1$,

$$
\gamma(t+1) g^{*}(t+1)=\left[\gamma(t) g^{*}(t)+\frac{d h}{d b}\left(b^{*}(t)\right)\right]^{+} .
$$

2) $b^{*}(t)<b^{*}(t-1)$ for $t=t_{1}, \ldots, T$. Moreover, for $t=$ $t_{1}, \ldots, T-1$,

$$
b^{*}(t)-b^{*}(t+1)>\frac{\gamma(t)}{\gamma(t+1)}\left(b^{*}(t-1)-b^{*}(t)\right) .
$$

The next result describes the behavior in the first phase of the second case of Theorem 1. If the optimal generation exceeds the demand at a certain time, then it must have stayed strictly above the demand at all previous times. Therefore the battery must have been in the charging mode throughout this period.

Lemma 3: Suppose A0 and A1 hold. If $b^{*}\left(t_{0}\right)<B$ and $g^{*}\left(t_{0}+1\right)>d\left(t_{0}+1\right)$ for some $t_{0} \in\{1, \ldots, T-1\}$ under an optimal policy, then the following hold.

1) $g^{*}(t)>d(t)$ for $t=1, \ldots, t_{0}+1$. Moreover, for $t=$ $1, \ldots, t_{0}$,

$$
\gamma(t+1) g^{*}(t+1)=\gamma(t) g^{*}(t)+\frac{d h}{d b}\left(b^{*}(t)\right) .
$$

2) $b^{*}(t)>b^{*}(t-1)$ for all $t=1, \ldots, t_{0}+1$. Moreover, for $t=2, \ldots, t_{0}+1$,

$$
b^{*}(t)-b^{*}(t-1)<\frac{\gamma(t-1)}{\gamma(t)}\left(b^{*}(t-1)-b^{*}(t-2)\right) .
$$

Lemmas 2 and 3 imply that the optimal generation $g^{*}(t)$ can cross $d(t)$ at most once, from above. Moreover, the optimal battery level is unimodal, i.e., it can only increase initially, until possibly reaching $B$. If the battery becomes 
saturated, it will remain saturated until the optimal generation drops below the demand, and can then only decrease for the remaining times. Case (1) in Theorem 1 describes the case where $b^{*}$ is never saturated and Case (2) in Theorem 1 describes the case where $b^{*}$ reaches saturation in phase two.

\section{NETWORK CASE}

We now consider a general network with multiple generators and multiple loads (i.e., the problem in (8)-(10)). To simplify, we eliminate the variables $q_{i}(t)$ by combining (2), (3), and (4) into

$$
\begin{aligned}
g_{i}(t)+r_{i}(t) & =\sum_{j \in \mathscr{N}} Y_{i j}\left(\theta_{i}(t)-\theta_{j}(t)\right), \quad i \in \mathscr{G}, \\
-d_{i}(t) & =\sum_{j \in \mathscr{N}} Y_{i j}\left(\theta_{i}(t)-\theta_{j}(t)\right), \quad i \in \mathscr{D} .
\end{aligned}
$$

Since the problem is convex, the KKT conditions are both necessary and sufficient. They imply the following characterization of the optimal generation

$$
g^{*}(t)=\Gamma^{-1}(t)\left[H^{*}(t)+B^{*}(t)\right]^{+}
$$

where

$$
\begin{aligned}
H^{*}(t) & =\operatorname{diag}\left(\sum_{\tau=t}^{T}-\frac{d h_{i}}{d b}\left(b_{i}^{*}(\tau)\right)\right) \\
B^{*}(t) & =\operatorname{diag}\left(\sum_{\tau=t}^{T}\left(\underline{b}_{i}^{*}(\tau)-\bar{b}_{i}^{*}(\tau)\right)\right)
\end{aligned}
$$

and $\Gamma(t)=\operatorname{diag}\left(\gamma_{i}(t)\right)$. Here, $b_{i}^{*}(t)$ is the optimal battery level at generator $i, \underline{b}_{i}^{*}(t)$ and $\bar{b}_{i}^{*}(t)$ are the Lagrange multipliers associated with $b_{i}^{*}(t)=0$ and $b_{i}^{*}(t)=B_{i}$ at generator $i$. The optimal generation given in (22) is a direct extension of (19) and (21) from the SGSL case to the network case. The effect of network is illustrated most prominently in the condition

$$
\left[\begin{array}{c}
g^{*}(t)+r^{*}(t) \\
-d(t)
\end{array}\right]=\mathbf{Y} \boldsymbol{\theta}^{*}(t)
$$

where the network admittance matrix $\mathbf{Y}$ is given by

$$
\mathbf{Y}_{i j}= \begin{cases}\sum_{k \in \mathscr{N}} Y_{i k}, & i=j \\ -Y_{i j}, & i \neq j .\end{cases}
$$

We expect the macroscopic structure of the optimal policy to generalize from SGSL to the network case. For example, from (22), since the marginal storage cost-to-go $H^{*}(t)$ decreases over time as long as $h_{i}^{\prime}\left(b_{i}\right)<0$, the optimal generation $g^{*}(t)$ tends to decrease over time as in the SGSL case. Battery reserves are used to meet demands toward the end of the horizon, relying more on power generation initially. However, unlike in the SGSL case where the generation and the demand are directly related, $g^{*}(t)+r^{*}(t)=d(t)$, in the network case, they are indirectly related through the admittance matrix and the voltage angles as shown in (23). This complicates the proof and the properties of optimal policy in the network case are under current study. Here, we present some numerical examples.

Example 2: Symmetric network with two generators. The network consists of two generators and 20 demand
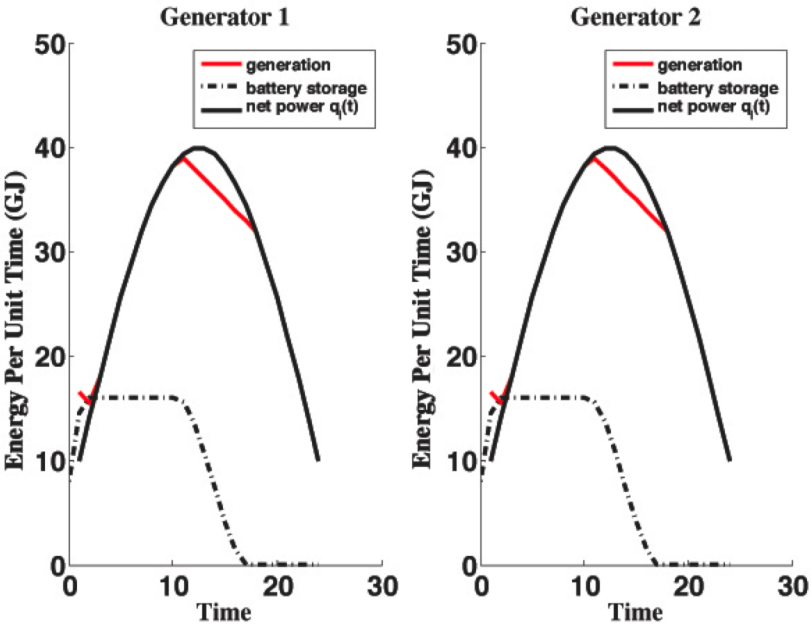

Fig. 4. Numerical results for the case with 2 generator nodes and 20 demand nodes. The configuration is symmetric, i.e. both generators are connected to every demand node, with equal admittance $Y_{i j}$ for every $i \in \mathscr{G}$ and $j \in \mathscr{D}$.

nodes. Every generator is directly connected to each of the demand nodes, but there is no direct connection between the two generators nor among the demand nodes. The admittance on every link is $Y_{i j}=1$. Link capacities $q_{i j}=1000 \mathrm{GJ}$ are the same for all $i, j . T=24$ hours, $B_{i}=16 \mathrm{GJ}$ and $b_{i}(0)=8 \mathrm{GJ}$. The load profile is $d_{i}(t)=3 \sin (\pi t / T)+1$ GJ. The storage cost is $h_{i}\left(b_{i}\right)=\alpha\left(B_{i}-b_{i}\right)$ with $\alpha=1 ; \gamma_{i}(t)=1$ in the generation cost function $c_{i}$.

The results are shown in Fig. 4. The optimal generation decreases when the battery initially charges and then tracks the net power output $q_{i}(t)$ as the battery remains saturated. The optimal generation then becomes strictly less than the net power output and the battery discharges until the battery drains completely and the generation again tracks net power output.

\section{Example 3: Cost savings.}

In this example, we consider both a SGSL case and a network case and illustrate how the time-varying nature of $\gamma_{i}(t)$ in the generation cost can affect the cost saving. The network that is used is similar to that in Example 2 except that the link capacities are not symmetric: some of the link capacities between generator 1 and the demand nodes are smaller. The behavior is shown in Fig. 5. Interestingly, the optimal battery energy $b^{*}(t)$ of generator 2 is not unimodal, i.e., it charges and discharges multiple times.

Without battery, the total generation must equal to total demand at each time. In particular, for SGSL, $g^{*}(t)=d(t)$ for all $t$ and the total cost is $\sum_{t} c(d(t), t)$ without battery. With battery, the generation cost can be reduced through optimization over time. Table I compares the optimal cost in (8) as a percentage of total cost without battery, for both single-generator-single-load and multi-generator-multiload (MGML) cases and for time-invariant and time-varying 

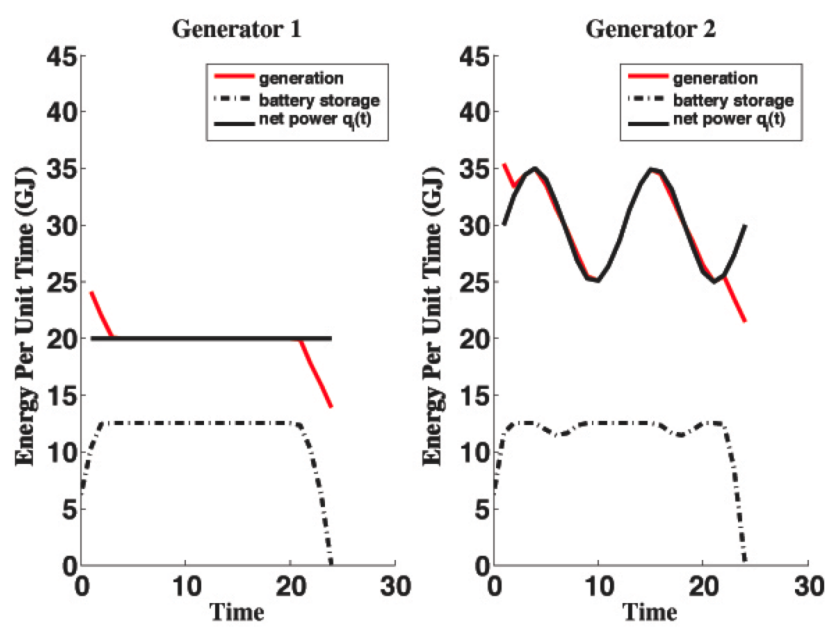

Fig. 5. Numerical results for the case with 2 generator nodes and 20 demand nodes with unequal link capacities. Demands vary sinusoidally.

TABLE I

OPTIMAL COSTS WITH BATTERIES AS \% OF TOTAL COST WITHOUT BATTERIES

\begin{tabular}{|c||c|c|}
\hline & SGSL & MGML \\
\hline time-invariant $\gamma_{i}$ & $98 \%$ & $97 \%$ \\
\hline time-varying $\gamma_{i}$ & $83 \%$ & $85 \%$ \\
\hline
\end{tabular}

$\gamma_{i}$. In the case with time-invariant $\gamma_{i}$, the cost saving is small. With time-varying $\gamma_{i}$, the cost savings in both the SGSL and the network cases are significantly higher. This confirms the intuition that battery is more valuable in the presence of fluctuations. The savings are greater in cases where the battery charges and discharges several times over the entire control period, which is a case that violates Assumption A1. Then with larger or more frequent fluctuations, an optimal policy has more opportunities to charge the batteries when generation costs are low and discharge when they are high.

\section{CONCLUSION}

In this paper, we have formulated a simple optimal power flow model with storage. We have characterized the optimal solution for the case with a single generator and a single load when the generation cost $c(g, t)$ is quadratic in $g$ and the battery cost $h(b)$ is strictly decreasing. In this case, under the assumption (A1) that the demand does not decrease too rapidly, the optimal generation schedule will cross the demand profile at most once, from above. The optimal battery level is unimodal where the battery is charged initially, possibly reaching saturation, and then discharges till the end of the control horizon. We have presented some numerical examples illustrating the behavior in the network case and when assumption A1 is not satisfied.

We plan to extend the analysis here in several ways. First, we will fully characterize the optimal solution in the network case by extending our results for the SGSL case. Second, both the demand profile and the power generation are deterministic in our current formulation, which is unrealistic. We will extend the current deterministic model to include randomness in demand and generation. Finally, we plan to incorporate a more realistic battery model that captures the dynamics of capacitance, as well as constraining the rate at which the battery can be charged or discharged.

\section{ACKNOWLEDGMENTS}

This work is partially supported by Southern California Edison (SCE), the Boeing Corporation, and the National Science Foundation. The authors wish to thank Christopher Clark, Jeff Gooding, and Percy Haralson of SCE for helpful discussions.

\section{REFERENCES}

[1] J. Carpentier, "Contribution to the economic dispatch problem," Bull. Soc. Franc. Electr., vol. 3, no. 8, pp. 431-447, 1962.

[2] B. Chowdhury and S. Rahman, "A review of recent advances in economic dispatch," IEEE Transactions on Power Systems, vol. 5, pp. $1248-1259,1990$

[3] M. Huneault and F. D. Galiana, "A survey of the optimal power flow literature," IEEE Transactions on Power Systems, vol. 6, no. 2, pp. 762-770, May 1991.

[4] J. A. Momoh, M. E. El-Hawary, and R. Adapa, "A review of selected optimal power flow literature to 1993, part i: Nonlinear and quadratic programming approaches," IEEE Transactions on Power Systems, vol. 14, no. 1, pp. 96-104, 1999.

[5] _ _A review of selected optimal power flow literature to 1993 , part ii: Newton, linear programming and interior point methods," IEEE Transactions on Power Systems, vol. 14, no. 1, pp. 105-111, 1999.

[6] K. S. Pandya and S. K. Joshi, "A survey of optimal power flow methods," Journal of Theoretical and Applied Information Technology, vol. 4, no. 5, pp. 450-458, 2008

[7] J. A. Momoh, Electric power system applications of optimization. Markel Dekker, 2001.

[8] "2007 Summer Loads and Resources Operations Assessment," California ISO, CA, Tech. Rep., 2007.

[9] "Californias electricity generation and transmission interconnection needs under alternative scenarios," Consultant Report, 2004.

[10] "Southern California Edison Signs Largest Wind Energy Contract in U.S. Renewable Industry History," Southern California Edison Press Release, 2006. [Online]. Available: http://www.edison.com/pressroom/pr.asp?id=6487

[11] C. Archer and M. Jacobson, "Evaluation of global wind power," Journal of Geophysical Research, vol. 110, no. D12110, 2005.

[12] T. Yau, L. Walker, H. Graham, and A. Gupta, "Effects of battery storage devices on power system dispatch," IEEE Transactions on Power Apparatus and Systems, vol. PAS-100, no. 1, pp. 375-383, 1981.

[13] N. Alguacil and A. J. Conejo, "Multiperiod optimal power flow using benders decomposition," IEEE Transactions on Power Systems, vol. 15, no. 1, pp. $196-201,2000$.

[14] E. Sortomme and M. A. El-Sharkawi, "Optimal power flow for a system of microgrids with controllable loads and battery storage," in Power Systems Conference and Exposition, 2009.

[15] M. Geidl and G. Andersson, "A modeling and optimization approach for multiple energy carrier power flow," in In Proc. of IEEE PES PowerTech, 2005.

[16] F. Wu, P. Varaiya, P. Spiller, and S. Oren, "Folk theorems on transmission access: Proofs and counterexamples," Journal of Regulatory Economics, vol. 10, no. 1, pp. 5-23, 1995.

[17] A. R. Bergen and V. Vittal, Power Systems Analysis, 2nd ed. Prentice Hall, 2000 